Rev. Mat. Iberoamericana 27 (2011), no. 3, 997-1022

\title{
Sub-Riemannian geometry of parallelizable spheres
}

\section{Mauricio Godoy Molina and Irina Markina}

\begin{abstract}
The first aim of the present paper is to compare various subRiemannian structures over the three dimensional sphere $S^{3}$ originating from different constructions. Namely, we describe the subRiemannian geometry of $S^{3}$ arising through its right action as a Lie group over itself, the one inherited from the natural complex structure of the open unit ball in $\mathbb{C}^{2}$ and the geometry that appears when it is considered as a principal $S^{1}$-bundle via the Hopf map. The main result of this comparison is that in fact those three structures coincide.

We present two bracket generating distributions for the seven dimensional sphere $S^{7}$ of step 2 with ranks 6 and 4 . The second one yields to a sub-Riemannian structure for $S^{7}$ that is not widely present in the literature until now. One of the distributions can be obtained by considering the CR geometry of $S^{7}$ inherited from the natural complex structure of the open unit ball in $\mathbb{C}^{4}$. The other one originates from the quaternionic analogous of the Hopf map.
\end{abstract}

\section{Introduction}

One of the main objectives of classical sub-Riemannian geometry is to study manifolds which are path-connected by curves admissible in a certain sense. In order to define what does admissibility mean in this context, we begin by setting notations that will be used throughout this paper. Let $M$ be a smooth connected manifold of dimension $n$, together with a smooth distribution $\mathcal{H} \subset T M$ of rank $2 \leq k<n$. Such vector bundles are often called horizontal in the literature. An absolutely continuous curve $\gamma:[0,1] \rightarrow M$ is called admissible or horizontal if $\dot{\gamma}(t) \in \mathcal{H}$ a.e.

2000 Mathematics Subject Classification: 53C17, 55R25, 32V15.

Keywords: Sub-Riemannian geometry, CR geometry, Hopf bundle, Ehresmann connection, parallelizable spheres, quaternions, octonions. 
Distributions satisfying the condition that their Lie-hull equals the whole tangent space of the manifold at each point play a central role in the search for horizontally path-connected manifolds. Such vector bundles are said to satisfy the bracket generating condition. To be more precise, define the following real vector bundles

$$
\mathcal{H}^{1}=\mathcal{H}, \quad \mathcal{H}^{r+1}=\left[\mathcal{H}^{r}, \mathcal{H}\right]+\mathcal{H}^{r} \quad \text { for } r \geq 1,
$$

which naturally give rise to the flag

$$
\mathcal{H}=\mathcal{H}^{1} \subseteq \mathcal{H}^{2} \subseteq \mathcal{H}^{3} \subseteq \cdots
$$

Then we say that a distribution is bracket generating if for all $x \in M$ there is an $r(x) \in \mathbb{Z}^{+}$such that

$$
\mathcal{H}_{x}^{r(x)}=T_{x} M
$$

If the dimensions $\operatorname{dim} \mathcal{H}_{x}^{r}$ do not depend on $x$ for any $r \geq 1$, we say that $\mathcal{H}$ is a regular distribution. The least $r$ such that (1.1) is satisfied is called the step of $\mathcal{H}$. We will focus on regular distributions of step 2. In [18] the reader can find detailed definitions and broad discussion about terminology.

The following classical result shows the precise relation between the notion of path-connectedness by means of horizontal curves and the assumption that $\mathcal{H}$ is a bracket generating distribution.

Theorem 1 ([11, 23]). Let $M$ be a connected manifold. If a distribution $\mathcal{H} \subset T M$ is bracket generating, then any two points in $M$ can be joined by a horizontal path.

We recall the definition of sub-Riemannian manifold.

Definition 1. A sub-Riemannian structure over a manifold $M$ is a pair $(\mathcal{H},\langle\cdot, \cdot\rangle)$, where $\mathcal{H}$ is a bracket generating distribution and $\langle\cdot, \cdot\rangle$ a fiber inner product defined on $\mathcal{H}$. In this setting, the length of an absolutely continuous horizontal curve $\gamma:[0,1] \rightarrow M$ is

$$
\ell(\gamma):=\int_{0}^{1}\|\dot{\gamma}(t)\| d t
$$

where $\|\dot{\gamma}(t)\|^{2}=\langle\dot{\gamma}(t), \dot{\gamma}(t)\rangle$ whenever $\dot{\gamma}(t)$ exists. The triple $(M, \mathcal{H},\langle\cdot, \cdot\rangle)$ is called sub-Riemannian manifold.

Thereby, restricting our considerations to connected sub-Riemannian manifolds endowed with bracket generating distributions, it is possible to define the notion of sub-Riemannian distance between two points. 
Definition 2. The sub-Riemannian distance $d(p, q) \in[0,+\infty)$ between two points $p, q \in M$ is given by $d(p, q):=\inf \ell(\gamma)$, where the infimum is taken over all absolutely continuous horizontal curves joining $p$ to $q$.

An absolutely continuous horizontal curve that realizes the distance between two points is called a horizontal length minimizer.

Remark: The connectedness of $M$ and the fact that $\mathcal{H}$ is bracket generating, assure that $d(p, q)$ is a finite nonnegative number. Nevertheless the bracket generating hypothesis, required for the previous definition, is possible to be weakened. In fact, in [25] the author finds a necessary and sufficient requirement to horizontal path-connectedness for a manifold in terms of the corresponding distribution. Clearly, this theorem contains, as a particular case, the bracket generating condition.

Historically, the first examples of sub-Riemannian manifolds that have been considered were Lie groups, see e.g. [2, 6, 9, 14, 17]. Due to its algebraic structure, it is sufficient to define appropriate distributions at the identity of the group. Right (or left) translations allow to find globally defined bracket generating distributions of right (or left) invariant vector fields. An important role has been played by considering domains in Euclidean spaces with special algebraic structures (such as the Heisenberg groups, $\mathbb{H}$-type groups as their natural generalizations to Clifford algebras, Engel groups, Carnot groups, etc.). Particular attention have had the three dimensional unimodular Lie groups which were studied, for example, in [2, 6, 14] and the Heisenberg group, see [13]. The main purpose of this communication is to present recent results concerning different sub-Riemannian structures of the second simplest family of examples of manifolds, namely, spheres. The main tool for the study of sub-Riemannian structures on spheres arise from the $G$-principal bundle structure given by the Hopf fibrations. We are also inspired by the article [26], where the close relation between the Hopf map and physical applications is presented.

The following celebrated theorem in topology, see [1], gives a very strong restriction on the problem of finding globally defined sub-Riemannian structures over spheres.

Theorem 2. (Adams). Let $S^{n-1}=\left\{x \in \mathbb{R}^{n}:\|x\|^{2}=1\right\}$ be the unit sphere in $\mathbb{R}^{n}$, with respect to the usual Euclidean norm $\|\cdot\|$. Then $S^{n-1}$ has precisely $\varrho(n)-1$ linearly independent, globally defined and non vanishing vector fields, where $\varrho(n)$ is defined in the following way: if $n=(2 a+1) 2^{b}$ and $b=c+4 d$ where $0 \leq c \leq 3$, then $\varrho(n)=2^{c}+8 d$.

In particular, two classical consequences follow: $S^{1}, S^{3}$ and $S^{7}$ are the only spheres with maximal number of linearly independent globally defined non vanishing vector fields, and the even dimensional spheres have no globally defined and non vanishing vector fields. 
The condition that a manifold $M$ has maximal number of linearly independent globally defined non vanishing vector fields is usually rephrased as saying that $M$ is parallelizable. The fact that $S^{1}, S^{3}$ and $S^{7}$ are the only parallelizable spheres was proved in [7] and that the even dimensional spheres have no globally defined and non vanishing vector fields is a consequence of the Hopf index theorem, see [27].

This theorem permits to conclude at least two major points of discussion: there is no possible global basis of a sub-Riemannian structure for spheres with even dimension and it is impossible to find a globally defined basis for bracket generating distributions, except for $S^{3}$ and $S^{7}$. The fact that $S^{3}$ and $S^{7}$ can be seen as the set of quaternions and octonions of unit length will play a core role in many arguments throughout this paper.

The main results that we present here are: a comparison between three sub-Riemannian structures of $S^{3}$ and the constructions for two different sub-Riemannian structures for $S^{7}$. More specifically, the first result can be summarized as an equivalence between the sub-Riemannian geometry of $S^{3}$ arising through its right Lie group action over itself as the set of unit quaternions, the one inherited from the natural complex structure of the open unit ball in $\mathbb{C}^{2}$ and the geometry that appears when considering the Hopf map as a principal $S^{1}$-bundle. Notice that this structure admits a tangent cone isomorphic to the one dimensional Heisenberg group in the sense of GromovMargulis-Mitchell-Mostow construction of the tangent cone [15, 20, 21, 22]. With respect to the second result, by considering the CR structure of $S^{7}$ inherited from the natural complex structure of the open unit ball in $\mathbb{C}^{4}$, we obtain a 2-step bracket generating distribution of rank 6 . This construction is intimately related to the Hopf fibration $S^{1} \rightarrow S^{7} \rightarrow \mathbb{C} P^{3}$, in the sense that the holomorphic tangent space defining the CR structure is an Ehresmann connection, that is, the orthogonal complement to the vertical space defined by the Hopf fibration as a principal $S^{1}$-bundle. This fact is generalized to all odd-dimensional spheres and, moreover, it implies that the tangent cone for $(2 n+1)$-dimensional spheres is isomorphic to the $n$-dimensional Heisenberg group. Making use of the quaternionic analogue of the Hopf map $S^{3} \rightarrow S^{7} \rightarrow S^{4}$, we present another 2-step bracket generating distribution that has rank 4 . We conclude that the sphere $S^{7}$ admits at least two different sub-Riemannian structures. The tangent cone, in the first case, is isomorphic to the 3-dimensional Heisenberg group, and in the second case it has the structure of the quaternionic Heisenberg-type group with 3-dimensional center [9]. In both cases we present the basis of the horizontal distribution that is very useful in future studies of geodesics and hypoelliptic operators related to the sub-Riemannian geometry of spheres. We would like to note that, in the case of the rank 6 distribution the given basis is globally defined, 
while in the case of the rank 4 distribution the search for a globally defined basis will be analyzed in a forthcoming paper. It is also expected that $S^{7}$ with the structure induced by the quaternionic Hopf fibration satisfies the conditions of a $\mathrm{qCR}$-manifold in the sense of [3].

\section{2. $S^{3}$ as a sub-Riemannian manifold}

Throughout this paper $\mathbb{H}$ will denote the quaternions, that is, $\mathbb{H}=\left(\mathbb{R}^{4},+, \circ\right)$ where + stands for the usual coordinate-wise addition in $\mathbb{R}^{4}$ and $\circ$ is a noncommutative product given by the formula

$$
\begin{aligned}
& \left(x_{0}+x_{1} i+x_{2} j+x_{3} k\right) \circ\left(y_{0}+y_{1} i+y_{2} j+y_{3} k\right)= \\
& =\left(x_{0} y_{0}-x_{1} y_{1}-x_{2} y_{2}-x_{3} y_{3}\right)+\left(x_{1} y_{0}+x_{0} y_{1}-x_{3} y_{2}+x_{2} y_{3}\right) i \\
& \quad+\left(x_{2} y_{0}+x_{3} y_{1}+x_{0} y_{2}-x_{1} y_{3}\right) j+\left(x_{3} y_{0}-x_{2} y_{1}+x_{1} y_{2}+x_{0} y_{3}\right) k .
\end{aligned}
$$

It is important to recall that $\mathbb{H}$ is a non-commutative, associative and normed real division algebra. Let $q=t+a i+b j+c k \in \mathbb{H}$, then the conjugate of $q$, is given by

$$
\bar{q}=t-a i-b j-c k .
$$

We define the norm $|q|$ of $q \in \mathbb{H}$ by $|q|^{2}=q \bar{q}$.

The realization of the sphere $S^{3}$ as the set of unit quaternions, produces a Lie group structure induced by quaternion multiplication.

The multiplication rule in $\mathbb{H}$ induces a right translation $R_{y}(x)$ of an element $x=x_{0}+x_{1} i+x_{2} j+x_{3} k$ by the element $y=y_{0}+y_{1} i+y_{2} j+y_{3} k$. The right invariant basis vector fields are defined as $Y(y)=\left(R_{y}(x)\right)_{*} Y(0)$, where $Y(0)$ are the basis vectors at the unity of the group. The matrix corresponding to the tangent map $\left(R_{y}(x)\right)_{*}$, obtained by the multiplication rule, becomes

$$
\left(R_{y}(x)\right)_{*}=\left(\begin{array}{cccc}
y_{0} & y_{1} & y_{2} & y_{3} \\
-y_{1} & y_{0} & -y_{3} & y_{2} \\
-y_{2} & y_{3} & y_{0} & -y_{1} \\
-y_{3} & -y_{2} & y_{1} & y_{0}
\end{array}\right)
$$

Calculating the action of $\left(R_{y}(x)\right)_{*}$ in the basis of unit vectors of $\mathbb{R}^{4}$ we get the four vector fields

$$
\begin{aligned}
& N(y)=y_{0} \partial_{y_{0}}+y_{1} \partial_{y_{1}}+y_{2} \partial_{y_{2}}+y_{3} \partial_{y_{3}}, \\
& V(y)=-y_{1} \partial_{y_{0}}+y_{0} \partial_{y_{1}}-y_{3} \partial_{y_{2}}+y_{2} \partial_{y_{3}}, \\
& X(y)=-y_{2} \partial_{y_{0}}+y_{3} \partial_{y_{1}}+y_{0} \partial_{y_{2}}-y_{1} \partial_{y_{3}}, \\
& Y(y)=-y_{3} \partial_{y_{0}}-y_{2} \partial_{y_{1}}+y_{1} \partial_{y_{2}}+y_{0} \partial_{y_{3}} .
\end{aligned}
$$


It is easy to see that $N(y)$ is the unit normal to $S^{3}$ at $y \in S^{3}$ with respect to the usual Riemannian structure $\langle\cdot, \cdot\rangle$ in $T \mathbb{R}^{4}$. Moreover, for any $y \in S^{3}$

$$
\langle N(y), V(y)\rangle_{y}=\langle N(y), X(y)\rangle_{y}=\langle N(y), Y(y)\rangle_{y}=0
$$

and

$$
\langle N(y), N(y)\rangle_{y}=\langle V(y), V(y)\rangle_{y}=\langle X(y), X(y)\rangle_{y}=\langle Y(y), Y(y)\rangle_{y}=1 .
$$

Since the matrix

$$
\left(\begin{array}{cccc}
-y_{1} & y_{0} & -y_{3} & y_{2} \\
-y_{2} & y_{3} & y_{0} & -y_{1} \\
-y_{3} & -y_{2} & y_{1} & y_{0}
\end{array}\right)
$$

has rank three, we conclude that the vector fields $\{V(y), X(y), Y(y)\}$ form an orthonormal basis of $T_{y} S^{3}$ with respect to $\langle\cdot, \cdot\rangle_{y}$, for any $y \in S^{3}$.

Observing that $[X, Y]=2 V$, we see that the distribution $\operatorname{span}\{X, Y\}$ is bracket generating, therefore it satisfies the hypothesis of Theorem 1 . The geodesics of the left invariant sub-Riemannian structure of $S^{3}$ are determined in [10], while in [17] the same results are achieved by considering the right invariant structure of $S^{3}$.

Notice that the distribution $\operatorname{span}\{X, Y\}$ can also be defined as the kernel of the contact one form

$$
\omega=-y_{1} d y_{0}+y_{0} d y_{1}-y_{3} d y_{2}+y_{2} d y_{3}
$$

Remark: It is easy to see that $[V, Y]=2 X$ and $[X, V]=2 Y$, therefore the distributions $\operatorname{span}\{Y, V\}$ and $\operatorname{span}\{X, V\}$ are also bracket generating. The corresponding contact forms are

$$
\theta=-y_{2} d y_{0}+y_{3} d y_{1}+y_{0} d y_{2}-y_{1} d y_{3}
$$

and

$$
\eta=-y_{3} d y_{0}-y_{2} d y_{1}+y_{1} d y_{2}+y_{0} d y_{3}
$$

respectively. This means that there is a priori no natural choice of a subRiemannian structure on $S^{3}$ generated by the Lie group action of multiplication of quaternions. Any choice that can be made, will produce essentially the same geometry. 


\section{3. $S^{3}$ as a CR manifold}

Consider $S^{3}$ as the boundary of the unit ball $B^{4}$ on $\mathbb{C}^{2}$, that is, as the hypersurface

$$
S^{3}:=\left\{(z, w) \in \mathbb{C}^{2}: z \bar{z}+w \bar{w}=1\right\} .
$$

The sphere $S^{3}$ cannot be endowed with a complex structure, but nevertheless it possess a differentiable structure compatible with the natural complex structure of the ball $B^{4}=\left\{(z, w) \in \mathbb{C}^{2}: z \bar{z}+w \bar{w}<1\right\}$ as an open set in $\mathbb{C}^{2}$. We will show that this differentiable structure over the sphere $S^{3}$ (CR structure) is equivalent to the sub-Riemannian one considered in the previous section. We begin by recalling the definition of a $\mathrm{CR}$ structure, according to $[5]$.

Definition 3. Let $W$ be a real vector space. A linear map $J: W \rightarrow W$ is called an almost complex structure map if $J \circ J=-I$, where $I: W \rightarrow W$ is the identity map.

In the case $W=T_{p} \mathbb{R}^{2 n}, p=\left(x_{1}, y_{1}, \ldots, x_{n}, y_{n}\right) \in \mathbb{R}^{2 n}$, we say that the standard almost complex structure for $W$ is defined by setting

$$
J_{n}\left(\partial_{x_{j}}\right)=\partial_{y_{j}}, \quad J_{n}\left(\partial_{y_{j}}\right)=-\partial_{x_{j}}, \quad 1 \leq j \leq n .
$$

For a smooth real submanifold $M$ of $\mathbb{C}^{n}$ and a point $p \in M$, in general the tangent space $T_{p} M$ is not invariant under the almost complex structure map $J_{n}$ for $T_{p} \mathbb{C}^{n} \cong T_{p} \mathbb{R}^{2 n}$. We are interested in the largest subspace invariant under the action of $J_{n}$.

Definition 4. For a point $p \in M$, the complex or holomorphic tangent space of $M$ at $p$ is the vector space

$$
H_{p} M=T_{p} M \cap J_{n}\left(T_{p} M\right) .
$$

In this setting, the following result takes place, see [5].

Lemma 1. Let $M$ be a real submanifold of $\mathbb{C}^{n}$ of real dimension $2 n-d$. Then

$$
2 n-2 d \leq \operatorname{dim}_{\mathbb{R}} H_{p} M \leq 2 n-d,
$$

and $\operatorname{dim}_{\mathbb{R}} H_{p} M$ is an even number.

A real submanifold $M$ of $\mathbb{C}^{n}$ is said to have a CR structure if $\operatorname{dim}_{\mathbb{R}} H_{p} M$ does not depend on $p \in M$. In particular, by Lemma 1 , every smooth real hypersurface $S$ embedded in $\mathbb{C}^{n}$ satisfies $\operatorname{dim}_{\mathbb{R}} H_{p} S=2 n-2$, therefore $S$ is a CR manifold. This fact applies to every odd dimensional sphere. 
The question addressed now is to describe $H_{p} S^{3}$. By the discussion in the previous paragraph, $H_{p} S^{3}$ can be seen as a complex vector space of complex dimension one. This description is achieved by considering the differential form

$$
\omega=\bar{z} d z+\bar{w} d w
$$

and observing that ker $\omega$ is precisely the set we are looking for. Straightforward calculations show that

$$
\operatorname{ker} \omega=\operatorname{span}\left\{\bar{w} \partial_{z}-\bar{z} \partial_{w}\right\} \text {. }
$$

In real coordinates this corresponds to

$$
\bar{w} \partial_{z}-\bar{z} \partial_{w}=\frac{1}{2}(-X+i Y)
$$

where $X$ and $Y$ were defined in Section 2. It is important to remark that this is precisely the maximal invariant $J_{2}$-subspace of $T_{p} S^{3}$, namely

$$
J_{2}(X)=Y, \quad J_{2}(Y)=-X,
$$

then $J_{2}(\operatorname{span}\{X, Y\})=\operatorname{span}\{X, Y\}$, but $J_{2}(V)=-N \notin T_{p} S^{3}$ for any point $p \in S^{3}$. Therefore, the distribution corresponding to the right invariant action of $S^{3}$ over itself is the same to its one dimensional (complex) CR structure.

Remark: The distribution associated to the anti-holomorphic form

$$
\bar{\omega}=z d \bar{z}+w d \bar{w}
$$

is the conjugate to the previous one and isomorphic to the 2-dimensional real distribution $\mathcal{H}$. More explicitly:

$$
\operatorname{ker} \omega=\operatorname{span}\left\{-w \partial_{\bar{z}}+z \partial_{\bar{w}}\right\}
$$

and in real coordinates this corresponds to

$$
-w \partial_{\bar{z}}+z \partial_{\bar{w}}=\frac{1}{2}(X+i Y) .
$$

The same almost complex structure as the previously described can be obtained by means of the covariant derivative of $S^{3}$ considered as a smooth Riemannian manifold embedded in $\mathbb{R}^{4}$. Namely, in [17] it is introduced the mapping $J(Z)=\nabla_{Z} V$, for $Z \in T S^{3}$, were $\nabla$ denotes the Levi-Civita connection on the tangent bundle to $S^{3}$ and $V$ is the vector field defined in Section 2. 


\section{4. $S^{3}$ as principal bundle}

In this section we describe how the structure of a principal $S^{1}$-bundle over $S^{3}$ induces a bracket generating distribution on $S^{3}$. Namely, it is possible to consider $S^{3}$ as a $S^{1}$-space, according to the action

$$
\lambda \cdot(z, w)=(\lambda z, \lambda w),
$$

where $\lambda \in S^{1}=\left\{\lambda \in \mathbb{C}:|\lambda|^{2}=1\right\}$ and $(z, w) \in S^{3}=\left\{(z, w) \in \mathbb{C}^{2}:\right.$ $\left.|z|^{2}+|w|^{2}=1\right\}$.

Consider the Hopf map $h: S^{3} \rightarrow S^{2}$ as a principal $S^{1}$-bundle, see [16, 19], given explicitly by

$$
h(z, w)=\left(|z|^{2}-|w|^{2}, 2 z \bar{w}\right),
$$

where $S^{2}=\left\{(x, \zeta) \in \mathbb{R} \times \mathbb{C}: x^{2}+|\zeta|^{2}=1\right\}$. Clearly, $h$ is a submersion of $S^{3}$ onto $S^{2}$, and it is a bijection between $S^{3} / S^{1}$ and $S^{2}$, where $S^{3} / S^{1}$ is understood as the orbit space of the $S^{1}$-action over $S^{3}$, previously defined.

Let $p=\left(x_{0}, \zeta_{0}\right) \in S^{2}$. It is easy to verify that $h^{-1}(p)=\left(z_{0}, w_{0}\right) \bmod S^{1}$, where $\left(z_{0}, w_{0}\right)$ is one preimage of $p$ under $h$. Consider the great circle

$$
\gamma_{p}(t)=e^{2 \pi i t}\left(z_{0}, w_{0}\right), \quad t \in[0,1],
$$

in $S^{3}$, that projects to $p$ under the Hopf map. Consider the tangent vector field, defined by

$$
\dot{\gamma}_{p}(t)=2 \pi i e^{2 \pi i t}\left(z_{0}, w_{0}\right) \in T_{\gamma_{p}(t)} S^{3} .
$$

We write the curve $\gamma_{p}$ and the map $d_{\gamma_{p}(t)} h$ in real coordinates, then

$$
\begin{aligned}
\gamma_{p}(t)=(z(t), w(t)) & =\left(x_{0}(t)+i x_{1}(t), x_{2}(t)+i x_{3}(t)\right) \\
& =\left(x_{0}(t), x_{1}(t), x_{2}(t), x_{3}(t)\right)
\end{aligned}
$$

and

$$
\left[d_{\gamma_{p}(t)} h\right]=2\left(\begin{array}{cccc}
x_{0}(t) & x_{1}(t) & -x_{2}(t) & -x_{3}(t) \\
x_{2}(t) & x_{3}(t) & x_{0}(t) & x_{1}(t) \\
-x_{3}(t) & x_{2}(t) & x_{1}(t) & -x_{0}(t)
\end{array}\right)
$$

Thus, the Hopf map induces the following action over the vector field $\dot{\gamma}_{p}(t)$ :

$$
\left[d_{\gamma_{p}(t)} h\right] \dot{\gamma}_{p}(t)=4 \pi\left(\begin{array}{rrrr}
x_{0}(t) & x_{1}(t) & -x_{2}(t) & -x_{3}(t) \\
x_{2}(t) & x_{3}(t) & x_{0}(t) & x_{1}(t) \\
-x_{3}(t) & x_{2}(t) & x_{1}(t) & -x_{0}(t)
\end{array}\right)\left(\begin{array}{c}
\dot{x}_{0}(t) \\
\dot{x}_{1}(t) \\
\dot{x}_{2}(t) \\
\dot{x}_{3}(t)
\end{array}\right)=\left(\begin{array}{l}
0 \\
0 \\
0 \\
0
\end{array}\right) \text {. }
$$


Therefore, if $\left[d_{\gamma_{p}(t)} h\right]$ is a full rank matrix, we would have characterized the kernel of it, by

$$
\text { ker } d_{\gamma_{p}(t)} h=\operatorname{span}\left\{\dot{\gamma}_{p}(t)\right\} \text {. }
$$

Notice that, using the notation of Section 2, the following identity holds

$$
\dot{\gamma}_{p}(t)=2 \pi V\left(\gamma_{p}(t)\right) .
$$

To see that the matrix $\left[d_{\gamma_{p}(t)} h\right]$ is full rank, observe that

$$
\left[d_{\gamma_{p}(t)} h\right]\left[d_{\gamma_{p}(t)} h\right]^{t}=4 I_{3}
$$

where $I_{3}$ denotes the identity matrix of size $3 \times 3$. This implies that $\left[d_{\gamma_{p}(t)} h\right]$ is full rank.

Before describing how the Hopf map induces a horizontal distribution, it is necessary to present some definitions found for example in [22, Chapter 11].

Definition 5. (Ehresmann Connection). Let $M$ and $Q$ be two differentiable manifolds, and let $\pi: Q \rightarrow M$ be a submersion. Denoting by $Q_{m}=\pi^{-1}(m)$ the fiber through $m \in M$, the vertical space at $q$ is the tangent space at the fiber $Q_{\pi(q)}$ and it is denoted by $V_{q}$.

An Ehresmann connection for the submersion $\pi: Q \rightarrow M$ is a distribution $\mathcal{H} \subset T Q$ which is everywhere transversal to the vertical, that is:

$$
V_{q} \oplus \mathcal{H}_{q}=T_{q} Q .
$$

We apply Definition 5 to the map $h$ in order to define the Ehresmann connection. Since we know that ker $d_{p} h=\operatorname{span}\{V(p)\}$, for every $p \in S^{3}$ by (4.1) and (4.2), and moreover,

$$
\langle X(p), V(p)\rangle_{p}=\langle Y(p), V(p)\rangle_{p}=\langle X(p), Y(p)\rangle_{p}=0,
$$

where $\langle\cdot, \cdot\rangle_{p}$ stands for the usual Riemannian structure defined at $p \in S^{3}$, we see that

$$
\mathcal{H}_{p}=\operatorname{span}\{X(p), Y(p)\}
$$

is an Ehresmann connection for the submersion $h: S^{3} \rightarrow S^{2}$ with $V(p)$ as a vertical space.

Definition 6. Let $G$ be a Lie group acting on $Q$ and $\pi: Q \rightarrow M$ a submersion, with Ehresmann connection $\mathcal{H}$, which is a fiber bundle with fiber $G$. The submersion $\pi$ is called a principal $G$-bundle with connection, if the following conditions hold:

- $G$ acts freely and transitively on fibers, 
- the group orbits are the fibers of $\pi: Q \rightarrow M$ (thus $M$ is isomorphic to $Q / G$ and $\pi$ is the canonical projection) and

- the $G$-action on $Q$ preserves the horizontal distribution $\mathcal{H}$;

We conclude that the Hopf fibration is a principal $S^{1}$-bundle with connection $\mathcal{H}$, defined by (4.3).

Definition 7. A sub-Riemannian metric $\langle\cdot, \cdot\rangle$ on the principal $G$-bundle $\pi: G \rightarrow M$ is called a metric of bundle type if the inner product $\langle\cdot, \cdot\rangle$ on the horizontal distribution $\mathcal{H}$ is induced from a Riemannian metric on $M$.

The sub-Riemannian metric $\left.\langle\cdot, \cdot\rangle\right|_{\mathcal{H}}$, obtained by restricting the usual Riemannian metric of $S^{3}$ to the distribution $\mathcal{H}$ is, by construction, a metric of bundle type.

Thus the Hopf map indicates, in a topological way, how to make a natural choice of the horizontal distribution $\mathcal{H}$ that was not obvious when we considered the right action of $S^{3}$ over itself.

Remark: Observe that the considered vector fields coincide with the right invariant vector fields. This phenomenon does not appear when we change the right action to the left action of $S^{3}$ over itself.

\section{Tangent vector fields for $S^{7}$}

In Sections 5 to 7 we will study different sub-Riemannian structures over the sphere $S^{7}$, using the ideas of Sections 2 to 4 . As a result, we obtain two structurally different types of horizontal distributions. One of them of rank 6 and other of the rank 4. Moreover, as we shall see, the sub-Riemannian structure induced by the CR structure and quaternionic analogue of the Hopf map are essentially different. We start from the construction of a convenient basis of tangent vector fields to $S^{7}$.

The multiplication of unit octonions is not associative, therefore $S^{7}$ is not a group in a contrast with $S^{3}$. Nevertheless, we still able to use the multiplication law in order to find global tangent vector fields. To do this, we present a multiplication table for the basis vectors of $\mathbb{R}^{8}$. This nonassociative multiplication gives rise to the division algebra of octonions

$$
\mathbb{O}=\operatorname{span}\left\{e_{0}, e_{1}, e_{2}, e_{3}, e_{4}, e_{5}, e_{6}, e_{7}\right\} .
$$

According to Table 1, the formula for the product of two octonions is presented in Subsection 8.1 of the Appendix. This multiplication rule induces a matrix representation of the right octonion multiplication, given 


\begin{tabular}{c||c|c|c|c|c|c|c|c} 
& $e_{0}$ & $e_{1}$ & $e_{2}$ & $e_{3}$ & $e_{4}$ & $e_{5}$ & $e_{6}$ & $e_{7}$ \\
\hline \hline$e_{0}$ & $e_{0}$ & $e_{1}$ & $e_{2}$ & $e_{3}$ & $e_{4}$ & $e_{5}$ & $e_{6}$ & $e_{7}$ \\
\hline$e_{1}$ & $e_{1}$ & $-e_{0}$ & $e_{3}$ & $-e_{2}$ & $e_{5}$ & $-e_{4}$ & $-e_{7}$ & $e_{6}$ \\
\hline$e_{2}$ & $e_{2}$ & $-e_{3}$ & $-e_{0}$ & $e_{1}$ & $e_{6}$ & $e_{7}$ & $-e_{4}$ & $-e_{5}$ \\
\hline$e_{3}$ & $e_{3}$ & $e_{2}$ & $-e_{1}$ & $-e_{0}$ & $e_{7}$ & $-e_{6}$ & $e_{5}$ & $-e_{4}$ \\
\hline$e_{4}$ & $e_{4}$ & $-e_{5}$ & $-e_{6}$ & $-e_{7}$ & $-e_{0}$ & $e_{1}$ & $e_{2}$ & $e_{3}$ \\
\hline$e_{5}$ & $e_{5}$ & $e_{4}$ & $-e_{7}$ & $e_{6}$ & $-e_{1}$ & $-e_{0}$ & $-e_{3}$ & $e_{2}$ \\
\hline$e_{6}$ & $e_{6}$ & $e_{7}$ & $e_{4}$ & $-e_{5}$ & $-e_{2}$ & $e_{3}$ & $-e_{0}$ & $-e_{1}$ \\
\hline$e_{7}$ & $e_{7}$ & $-e_{6}$ & $e_{5}$ & $e_{4}$ & $-e_{3}$ & $-e_{2}$ & $e_{1}$ & $-e_{0}$ \\
\hline
\end{tabular}

Table 1: Multiplication table for the basis of $\mathbb{O}$.

explicitly by:

$$
\left(R_{y}(x)\right)_{*}=\left(\begin{array}{cccccccc}
y_{0} & -y_{1} & -y_{2} & -y_{3} & -y_{4} & -y_{5} & -y_{6} & -y_{7} \\
y_{1} & y_{0} & y_{3} & -y_{2} & y_{5} & -y_{4} & -y_{7} & y_{6} \\
y_{2} & -y_{3} & y_{0} & y_{1} & y_{6} & y_{7} & -y_{4} & -y_{5} \\
y_{3} & y_{2} & -y_{1} & y_{0} & y_{7} & -y_{6} & y_{5} & -y_{4} \\
y_{4} & -y_{5} & -y_{6} & -y_{7} & y_{0} & y_{1} & y_{2} & y_{3} \\
y_{5} & y_{4} & -y_{7} & y_{6} & -y_{1} & y_{0} & -y_{3} & y_{2} \\
y_{6} & y_{7} & y_{4} & -y_{5} & -y_{2} & y_{3} & y_{0} & -y_{1} \\
y_{7} & -y_{6} & y_{5} & y_{4} & -y_{3} & -y_{2} & y_{1} & y_{0}
\end{array}\right) .
$$

We are able to find globally defined tangent vector fields which are invariant under the right product rule. We proceed by analogy with Section 2 . The explicit formulae are given in Subsection 8.2 of the Appendix. The vector fields $\left\{Y_{0}, \ldots, Y_{7}\right\}$ form a frame for $T \mathbb{R}^{8}$ and, as in Subsection 8.2, the vector fields $\left\{Y_{1}, \ldots, Y_{7}\right\}$ form a frame for $T S^{7}$. More explicitly, we have that the following identities hold

$$
\left\langle Y_{i}(y), Y_{j}(y)\right\rangle_{y}=\delta_{i j}, \quad y \in S^{7}, \quad i, j \in\{0,1, \ldots, 7\}
$$

where $\langle\cdot, \cdot\rangle$ is the standard Riemannian structure over $\mathbb{R}^{8}$ and $\delta_{i j}$ stands for Kronecker's delta.

Remark: Recall that in contrast with quaternions, the matrix representation $\left(R_{y}(x)\right)_{*}$ of right octonion multiplication is only a convenient way of writing the formula presented in Subsection 8.1 of the Appendix. For quaternions this is actually a representation of quaternion product, but it cannot be such for octonions since they are non-associative and matrix multiplication is associative. 


\section{CR structure and the Hopf map on $S^{7}$}

In [22, Chapter 11] it is briefly discussed the general idea of studying a sub-Riemannian geometry for odd dimensional spheres via the higher Hopf fibrations. Namely, consider $S^{2 n+1}=\left\{z \in \mathbb{C}^{n+1}:\|z\|^{2}=1\right\}$, then the $S^{1}$-action on $S^{2 n+1}$ given by

$$
\lambda \cdot\left(z_{0}, \ldots, z_{n}\right)=\left(\lambda z_{0}, \ldots, \lambda z_{n}\right),
$$

for $\lambda \in S^{1}$ and $\left(z_{0}, \ldots, z_{n}\right) \in S^{2 n+1}$, induces the well-known principal $S^{1}$-bundle

$$
S^{1} \rightarrow S^{2 n+1} \stackrel{H}{\longrightarrow} \mathbb{C} P^{n}
$$

given explicitly by

$$
S^{2 n+1} \ni\left(z_{0}, \ldots, z_{n}\right) \mapsto H\left(z_{0}, \ldots, z_{n}\right)=\left[z_{0}: \cdots: z_{n}\right] \in \mathbb{C} P^{n},
$$

where $\left[z_{0}: \cdots: z_{n}\right]$ denotes homogeneous coordinates. This map is called higher Hopf fibration. The kernel of the map $h: S^{2 n+1} \rightarrow \mathbb{C} P^{n}$ produces the vertical space and a transversal to the vertical space distribution gives the Ehresmann connection. We show that the vertical space is always given by an action of standard almost complex structure on the normal vector field to $S^{2 n+1}$, and the Ehresmann connection coincides with the holomorphic tangent space at each point of $S^{2 n+1}$.

Theorem 2 asserts that any odd dimensional sphere has at least one globally defined non vanishing tangent vector field. If the dimension of the sphere is of the form $4 n+1$, then it has only one globally defined non vanishing tangent vector field. In the case that the dimension of the sphere is of the form $4 n+3$, then the sphere admits at least three globally defined non vanishing vector fields. Any sphere $S^{2 n+1}$ possesses the vector field

$$
V_{n+1}(y)=-y_{1} \partial_{y_{0}}+y_{0} \partial_{y_{1}}-y_{3} \partial_{y_{2}}+\cdots-y_{2 n+2} \partial_{y_{2 n+1}}+y_{2 n+1} \partial_{y_{2 n+2}} .
$$

Observe that this vector field has appeared already in two opportunities: the vector field $V$ in Sections 2, 3 and 4 corresponds to $V_{2}$; and the vector field $Y_{1}$ in Subsection 8.2 of the Appendix corresponds to $V_{4}$.

The vector field $V_{n+1}$ encloses valuable information concerning the CR structure of $S^{2 n+1}$. We know by Lemma 1 that, as a smooth hypersurface in $\mathbb{C}^{n+1}$ the sphere $S^{2 n+1}$ admits a holomorphic tangent space of dimension

$$
\operatorname{dim}_{\mathbb{R}} H_{p} S^{2 n+1}=2 n
$$

for any point $p \in S^{2 n+1}$. The following lemma implies the description of $H_{p} S^{2 n+1}$ as the orthogonal complement to $V_{n+1}$. 
Lemma 2. Let $W$ be an Euclidean space of dimension $n+2, n \geq 1$, and inner product $\langle\cdot, \cdot\rangle_{W}$. Consider an orthogonal decomposition $W=$ $\operatorname{span}\{X, Y\} \oplus_{\perp} \widetilde{W}$ with respect to $\langle\cdot, \cdot\rangle_{W}$ and an orthogonal endomorphism $A: W \rightarrow W$ such that

$$
A(\operatorname{span}\{X, Y\})=\operatorname{span}\{X, Y\},
$$

then $\widetilde{W}$ is an invariant space under the action of $A$, i.e.

$$
A(\widetilde{W})=\widetilde{W}
$$

Proof. Let $v \in \widetilde{W}$, then for any $\alpha, \beta \in \mathbb{R}$ it is clear that

$$
\langle A v, \alpha X+\beta Y\rangle_{W}=\left\langle v, A^{t}(\alpha X+\beta Y)\right\rangle_{W}=\left\langle v, A^{-1}(\alpha X+\beta Y)\right\rangle_{W} .
$$

Since $A(\operatorname{span}\{X, Y\})=\operatorname{span}\{X, Y\}$, there exist $a, b \in \mathbb{R}$ such that

$$
A^{-1}(\alpha X+\beta Y)=a X+b Y
$$

and therefore

$$
\langle A v, \alpha X+\beta Y\rangle_{W}=\langle v, a X+b Y\rangle_{W}=0,
$$

which implies that $A v \in \widetilde{W}$.

As an application of Lemma 2, it is possible to obtain the explicit characterization of the previously mentioned space $H_{p} S^{2 n+1}$.

Lemma 3. The vector space $H_{p} S^{2 n+1}$ is the orthogonal complement to the vector $V_{n+1}(p)$ in $T_{p} S^{2 n+1}$, for any $p \in S^{2 n+1}$.

Proof. Consider the vector space

$$
W_{p}=\operatorname{span}\left\{N_{n+1}(p)\right\} \oplus_{\perp} T_{p} S^{2 n+1} \cong T_{p} \mathbb{R}^{2 n+2},
$$

where $N_{n+1}(p)$ is the normal vector to $S^{2 n+1}$ at $p$. The standard almost complex structure map $J_{n+1}: W_{p} \rightarrow W_{p}$ is orthogonal. Moreover

$$
J_{n+1}\left(V_{n+1}(p)\right)=-N_{n+1}(p), \quad J_{n+1}\left(N_{n+1}(p)\right)=V_{n+1}(p) .
$$

Using the decomposition $W_{p}=\widetilde{W}_{p} \oplus_{\perp} \operatorname{span}\left\{V_{n+1}(p), N_{n+1}(p)\right\}$, it is possible to apply Lemma 2 in order to conclude that $\widetilde{W}_{p}$, which is the orthogonal complement to $V_{n+1}(p)$ in $T_{p} S^{2 n+1}$, is invariant under $J_{n+1}$. Since $\operatorname{dim}_{\mathbb{R}} \widetilde{W}_{p}=2 n$, we conclude that $\widetilde{W}_{p}=H_{p} S^{2 n+1}$. 
Remark: The space $H S^{2 n+1}$ can also be described as the kernel of the one-form

$$
\theta_{n+1}=\bar{z}_{0} d z_{0}+\ldots+\bar{z}_{n} d z_{n} .
$$

Indeed, consider $X \in H S^{2 n+1}$, then by straightforward calculations we have

$$
\theta_{n+1}(X)=\left\langle X, N_{n+1}\right\rangle+i\left\langle X, V_{n+1}\right\rangle=0 .
$$

Lemma 3 provides a horizontal distribution of rank $2 n$ for the spheres $S^{2 n+1}$, by considering the holomorphic tangent space. The goal now is to prove that this distribution is bracket generating. In order to do this, let us state a simple result establishing the bracket generating property for an arbitrary contact manifold.

Lemma 4. Let $M$ be a $(2 n+1)$-dimensional contact manifold with contact form $\omega$, then $\xi=\operatorname{ker} \omega$ is a bracket generating distribution of rank $2 n$ and step 2 .

Proof. Recall Cartan's formula for a differential one-form $\omega$, namely

$$
d \omega(X, Y)=X(\omega(Y))-Y(\omega(X))-\omega([X, Y]),
$$

for all $X, Y \in T M$. See [8] for the general formulation. It follows from (6.2) that $\xi$ is Frobenius integrable if and only if $d \omega(X, Y)=0$ for all $X, Y \in \xi$. Thus, if $\omega$ is a contact form, then $d \omega(X, Y) \neq 0$ for all $X, Y \in T M$ and, therefore $\xi$ is not Frobenius integrable. This implies the bracket generating property for $\xi$, since if $[X, Y](p) \notin \xi_{p}$ at any point $p \in M$ for some $X(p), Y(p) \in \xi_{p}$ then $\operatorname{span}\{[X, Y](p)\} \oplus \xi_{p}=T_{p} M$.

By Lemma 4, to prove that $H S^{2 n+1}$ is bracket generating, it is sufficient to find a contact one-form $\omega_{n+1}$ such that $H S^{2 n+1}=\operatorname{ker} \omega_{n+1}$. In order to achieve this, consider

$$
\omega_{n+1}=\operatorname{Im} \theta_{n+1}=-y_{1} d y_{0}+y_{0} d y_{1}-\ldots-y_{2 n+1} d y_{2 n}+y_{2 n} d y_{2 n+1}
$$

defined on $S^{2 n+1}$. By (6.1), the relation $H S^{2 n+1}=\operatorname{ker} \omega_{n+1}$ holds immediately.

Theorem 3. The one-form $\omega_{n+1}$ defined in (6.3) is a contact form. More specifically, $\omega_{n+1}$ satisfies

$$
\left(d \omega_{n+1}\right)^{n} \wedge \omega_{n+1}=n ! \cdot 2^{n} \operatorname{dvol}_{S^{2 n+1}},
$$

where $\mathrm{dvol}_{S^{2 n+1}}$ is the volume form for $S^{2 n+1}$. 
Proof. We observe that

$$
d \omega_{n+1}=2\left(d y_{0} \wedge d y_{1}+\cdots+d y_{2 n} \wedge d y_{2 n+1}\right) .
$$

Now, recalling the multinomial formula

$$
\left(x_{1}+\ldots+x_{m}\right)^{p}=\sum_{i_{1}+\cdots+i_{m}=p}\left(\begin{array}{c}
p \\
i_{1} \ldots i_{m}
\end{array}\right) x_{1}^{i_{1}} \cdots x_{m}^{i_{m}},
$$

where $\left(\begin{array}{c}p \\ i_{1} \ldots i_{m}\end{array}\right)$ denotes the multinomial coefficient $\frac{p !}{i_{1} ! \cdots i_{m} !}$. Then

$$
\begin{aligned}
& \left(d \omega_{n+1}\right)^{n}=2^{n} \sum_{i_{0}+\cdots+i_{n}=n}\left(\begin{array}{c}
n \\
i_{0} \ldots i_{n}
\end{array}\right)\left(d y_{0} \wedge d y_{1}\right)^{i_{0}} \wedge \cdots \wedge\left(d y_{2 n} \wedge d y_{2 n+1}\right)^{i_{n}} \\
& =n ! \cdot 2^{n} \sum_{j=0}^{n}\left(d y_{0} \wedge d y_{1}\right) \wedge \cdots \wedge\left(\widehat{d y_{2 j}} \wedge \widehat{d y_{2 j+1}}\right) \wedge \cdots \wedge\left(d y_{2 n} \wedge d y_{2 n+1}\right)
\end{aligned}
$$

where $\widehat{d y}_{k}$ means that this term is ommited. The fact that the differential one-forms are grouped in pairs in (6.4), permits us to use the multinomial formula. Equality (6.5) holds since in the summation the only non-zero terms are those when $i_{0}, \ldots, i_{n} \in\{0,1\}$ and $i_{0}+\cdots+i_{n}=n$. In this case

$$
\left(\begin{array}{c}
n \\
i_{0} \ldots i_{n}
\end{array}\right)=\frac{n !}{0 ! \cdot 1 ! \cdots 1 !}=n !
$$

Taking the exterior power of $\omega_{n+1}$ and expression (6.5) we see that

$$
\begin{aligned}
& \left(d \omega_{n+1}\right)^{n} \wedge \omega_{n+1}=n ! \cdot 2^{n} \sum_{j=0}^{2 n+1}(-1)^{j} y_{j} d y_{0} \wedge \cdots \wedge{\widehat{d y_{j}}} \wedge \wedge d y_{2 n+1} \\
& =n ! \cdot 2^{n} \operatorname{dvol}_{S^{2 n+1}} \text {. }
\end{aligned}
$$

The following corollary holds, by Lemma 4 and Theorem 3.

Corollary 1. The holomorphic tangent bundle $H S^{2 n+1}$ is a bracket generating distribution of step 2 and rank $2 n$.

An important consequence of Theorem 3 follows by considering a classical result by G. Darboux, see [12]. In modern terms, this theorem asserts that every $(2 n+1)$-dimensional contact manifold is locally the $n$-dimensional Heisenberg group. This means precisely that the tangent cone of $S^{2 n+1}$ as a sub-Riemannian manifold with distribution $H S^{2 n+1}$ and metric induced by the usual Euclidean metric in $\mathbb{R}^{2 n+2}$ is isomorphic to the $n$-dimensional Heisenberg group.

It is necessary to remark that in general there is no globally defined basis for $H S^{2 n+1}$. By Theorem 2, this is only possible for $S^{3}$ and $S^{7}$. A basis for this distribution in the case of $S^{3}$ was already discussed in Section 2. Here we present an explicit proof that shows the bracket generating property of 
the basis of $H S^{7}$ invariant under right octonion multiplication. A similar proof and other considerations concerning the hypoelliptic nature of the sub-Laplacian associated with the distribution $H S^{7}$ can be found in [4].

Theorem 4. The subbundle $\mathcal{H}=\operatorname{span}\left\{Y_{2}, \ldots, Y_{7}\right\}=H S^{7}$ of $T S^{7}$ is a bracket generating distribution of rank 6 and step 2.

Proof. Define the following vector fields

$$
\begin{aligned}
& v_{41}(y)=-y_{4} \partial_{y_{0}}+y_{5} \partial_{y_{1}}+y_{0} \partial_{y_{4}}-y_{1} \partial_{y_{5}}, \\
& v_{42}(y)=y_{6} \partial_{y_{2}}-y_{7} \partial_{y_{3}}-y_{2} \partial_{y_{6}}+y_{3} \partial_{y_{7}}, \\
& v_{51}(y)=-y_{5} \partial_{y_{0}}-y_{4} \partial_{y_{1}}+y_{1} \partial_{y_{4}}+y_{0} \partial_{y_{5}}, \\
& v_{52}(y)=-y_{7} \partial_{y_{2}}-y_{6} \partial_{y_{3}}+y_{3} \partial_{y_{6}}+y_{0} \partial_{y_{7}},
\end{aligned}
$$

and observe that $v_{41}+v_{42}=Y_{4}$ and $v_{51}+v_{52}=Y_{5}$. By straightforward calculations we see that

$$
\begin{aligned}
& \left\langle v_{41}(y), Y_{0}(y)\right\rangle_{y}=\left\langle v_{42}(y), Y_{0}(y)\right\rangle_{y}=\left\langle v_{51}(y), Y_{0}(y)\right\rangle_{y}=\left\langle v_{52}(y), Y_{0}(y)\right\rangle_{y}=0, \\
& \left\langle v_{41}(y), Y_{1}(y)\right\rangle_{y}=\left\langle v_{42}(y), Y_{1}(y)\right\rangle_{y}=\left\langle v_{51}(y), Y_{1}(y)\right\rangle_{y}=\left\langle v_{52}(y), Y_{1}(y)\right\rangle_{y}=0,
\end{aligned}
$$

which implies that $v_{41}, v_{42}, v_{51}, v_{52} \in \operatorname{span}\left\{Y_{2}, \ldots, Y_{7}\right\}$. The following commutation relation

$$
\left[v_{41}, v_{51}\right]+\left[v_{42}, v_{52}\right]=-2 Y_{1}
$$

implies that the distribution $\mathcal{H}$ is bracket generating of step 2 .

Remark: It is possible to repeat the previous argument with other pairs of vector fields. For example, if instead of $Y_{4}$ and $Y_{5}$ we employ $Y_{2}$ and $Y_{3}$, we can consider the vector fields

$$
\begin{aligned}
& v_{21}(y)=-y_{2} \partial_{y_{0}}+y_{3} \partial_{y_{1}}+y_{0} \partial_{y_{2}}-y_{1} \partial_{y_{3}}, \\
& v_{22}(y)=-y_{6} \partial_{y_{4}}+y_{7} \partial_{y_{5}}+y_{4} \partial_{y_{6}}-y_{5} \partial_{y_{7}}, \\
& v_{31}(y)=-y_{3} \partial_{y_{0}}-y_{2} \partial_{y_{1}}+y_{1} \partial_{y_{2}}+y_{0} \partial_{y_{3}}, \\
& v_{32}(y)=y_{7} \partial_{y_{4}}+y_{6} \partial_{y_{5}}-y_{5} \partial_{y_{6}}-y_{4} \partial_{y_{7}},
\end{aligned}
$$

satisfy $v_{21}+v_{22}=Y_{2}, v_{31}+v_{32}=Y_{3}$ and

$$
\left[v_{21}, v_{31}\right]-\left[v_{21}, v_{31}\right]=-2 Y_{1}
$$

We can proceed in a similar way if we use $Y_{6}$ and $Y_{7}$.

We conclude this section by proving that the line bundle $\operatorname{span}\left\{V_{n+1}\right\}$ is the vertical space for the submersion given by the Hopf fibration $S^{1} \rightarrow$ $S^{2 n+1} \stackrel{H}{\longrightarrow} \mathbb{C} P^{n}$. This implies that the distribution $\mathcal{H}$ defined in Theorem 4 
is an Ehresmann connection for $H$. To achieve this, we recall that the charts defining the holomorphic structure of $\mathbb{C} P^{n}$ are given by the open sets

$$
U_{k}=\left\{\left[z_{0}: \cdots: z_{n}\right]: z_{k} \neq 0\right\},
$$

together with the homeomorphisms

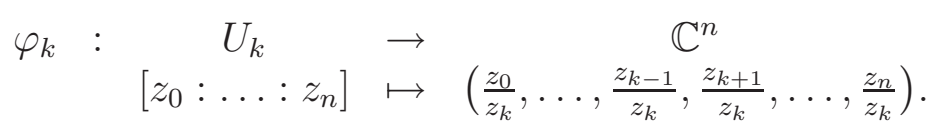

Then, without loss of generality we will assume that $n=3$ and we will develop the explicit calculations for $k=0$. The other cases can be treated similarly.

Using the chart $\left(U_{0}, \varphi_{0}\right)$ defined above, we have the map

$$
\begin{aligned}
& \varphi_{0} \circ H \quad: \quad S^{7} \quad \rightarrow \quad \mathbb{C}^{3} \\
& \left(z_{0}, z_{1}, z_{2}, z_{3}\right) \mapsto\left(\frac{z_{1}}{z_{0}}, \frac{z_{2}}{z_{0}}, \frac{z_{3}}{z_{0}}\right),
\end{aligned}
$$

which in real coordinates can be written as

$$
\begin{aligned}
\varphi_{0} \circ H\left(x_{0}, \ldots, x_{7}\right)=( & \frac{x_{0} x_{2}+x_{1} x_{3}}{x_{0}^{2}+x_{1}^{2}}, \frac{x_{0} x_{3}-x_{1} x_{2}}{x_{0}^{2}+x_{1}^{2}}, \frac{x_{0} x_{4}+x_{1} x_{5}}{x_{0}^{2}+x_{1}^{2}}, \\
& \left.\frac{x_{0} x_{5}-x_{1} x_{4}}{x_{0}^{2}+x_{1}^{2}}, \frac{x_{0} x_{6}+x_{1} x_{7}}{x_{0}^{2}+x_{1}^{2}}, \frac{x_{0} x_{7}-x_{1} x_{6}}{x_{0}^{2}+x_{1}^{2}}\right) .
\end{aligned}
$$

The differential of this mapping is given by the matrix
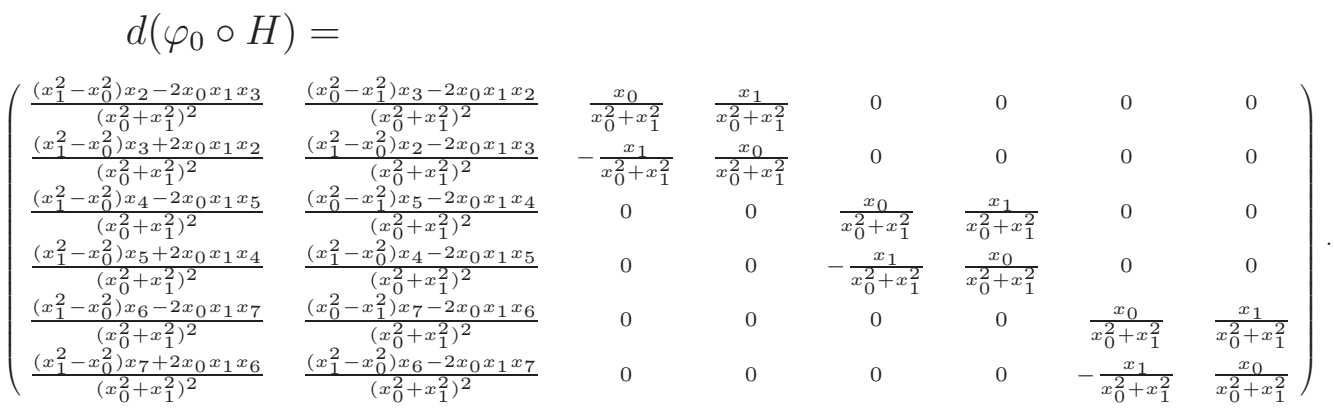

By straightforward calculations, we know that

$$
\operatorname{det}\left(\left[d\left(\varphi_{0} \circ H\right)\right]\left[d\left(\varphi_{0} \circ H\right)\right]^{t}\right)=\left(x_{0}^{2}+x_{1}^{2}\right)^{-8}=\left|z_{0}\right|^{-16} \neq 0 \text {, }
$$

therefore, the matrix $d\left(\varphi_{0} \circ H\right)$ has rank 6 or equivalently:

$$
\operatorname{dim}_{\mathbb{R}} \operatorname{ker} d\left(\varphi_{0} \circ H\right)=2 \text {. }
$$

Moreover, since

$$
d\left(\varphi_{0} \circ H\right)\left(N_{n+1}\right)=d\left(\varphi_{0} \circ H\right)\left(V_{n+1}\right)=0,
$$

by direct calculations, we conclude

$$
\operatorname{ker} d\left(\varphi_{0} \circ H\right)=\operatorname{span}\left\{N_{n+1}, V_{n+1}\right\} \text {. }
$$

This implies that ker $d H=\operatorname{span}\left\{V_{n+1}\right\}$. 


\section{Application of the first quaternionic Hopf map}

Trying to imitate the work already done for $S^{3}$, we find through the quaternionic Hopf bundle $S^{3} \rightarrow S^{7} \rightarrow S^{4}$ a natural choice of horizontal distributions. We consider the quaternionic Hopf map given by

$$
h: \begin{array}{ccc}
S^{7} & \rightarrow & S^{4} \\
(z, w) & \mapsto & \left(|z|^{2}-|w|^{2}, 2 z \bar{w}\right)
\end{array},
$$

which can be written in real coordinates as:

$$
\begin{aligned}
& h\left(x_{0}, \ldots, x_{7}\right)=\left(x_{0}^{2}+x_{1}^{2}+x_{2}^{2}+x_{3}^{2}-x_{4}^{2}-x_{5}^{2}-x_{6}^{2}-x_{7}^{2},\right. \\
& 2\left(x_{0} x_{4}+x_{1} x_{5}+x_{2} x_{6}+x_{3} x_{7}\right), 2\left(-x_{0} x_{5}+x_{1} x_{4}-x_{2} x_{7}+x_{3} x_{6}\right), \\
& \left.2\left(-x_{0} x_{6}+x_{1} x_{7}+x_{2} x_{4}-x_{3} x_{5}\right), 2\left(-x_{0} x_{7}-x_{1} x_{6}+x_{2} x_{5}+x_{3} x_{4}\right)\right) .
\end{aligned}
$$

The differential map $d h$ is the following:

$$
d h=2\left(\begin{array}{cccccccc}
x_{0} & x_{1} & x_{2} & x_{3} & -x_{4} & -x_{5} & -x_{6} & -x_{7} \\
x_{4} & x_{5} & x_{6} & x_{7} & x_{0} & x_{1} & x_{2} & x_{3} \\
-x_{5} & x_{4} & -x_{7} & x_{6} & x_{1} & -x_{0} & x_{3} & -x_{2} \\
-x_{6} & x_{7} & x_{4} & -x_{5} & x_{2} & -x_{3} & -x_{0} & x_{1} \\
-x_{7} & -x_{6} & x_{5} & x_{4} & x_{3} & x_{2} & -x_{1} & -x_{0}
\end{array}\right) .
$$

Since none of the commutators $\left[Y_{i}, Y_{j}\right], i, j=1, \ldots, 7$ coincides with the $Y_{k}, k=1, \ldots, 7$, we look for the kernel of $d h$ among the commutators $Y_{i j}$, $i, j=1, \ldots, 7$. We found that $[d h] Y_{45}=[d h] Y_{46}=[d h] Y_{56}=0$. Define $V=\left\{Y_{45}, Y_{46}, Y_{56}\right\}$.

Our next step is to find the horizontal distribution $\operatorname{span}\{\mathcal{H}\}$ that is transversal to $\operatorname{span}\{V\}$ and bracket generating: $\operatorname{span}\{\mathcal{H}\}_{p} \oplus \operatorname{span}\{V\}_{p}=$ $T_{p} S^{7}$ for all $p \in S^{7}$. To begin with we define five basis for horizontal distributions, that we will work with

$$
\begin{array}{ll}
\mathcal{H}_{0}=\left\{Y_{47}, Y_{57}, Y_{67}, W\right\}, & \\
\mathcal{H}_{1}=\left\{Y_{34}, Y_{35}, Y_{36}, Y_{37}\right\}, & \mathcal{H}_{2}=\left\{Y_{24}, Y_{25}, Y_{26}, Y_{27}\right\}, \\
\mathcal{H}_{3}=\left\{Y_{14}, Y_{15}, Y_{16}, Y_{17}\right\}, & \mathcal{H}_{4}=\left\{Y_{04}, Y_{05}, Y_{06}, Y_{07}\right\},
\end{array}
$$

where the vector field $W$ will be defined later and the notation $Y_{0 k}=Y_{k}$ is chosen for convenience. The numeration is valid only for this section.

We collect some useful information about sets $\mathcal{H}_{m}, m=0, \ldots, 4$, that we will exploit later.

1. All vector fields inside $\mathcal{H}_{m}, m=0,1,2,3,4$ are orthonormal (we do not count $W$ before we precise it). 
2. All of collections $\mathcal{H}_{m}, m=0,1,2,3,4$ are bracket generating with the following commutator relations:

$$
\begin{aligned}
& \frac{1}{2}\left[Y_{j 4}, Y_{j 5}\right]=Y_{45}, \frac{1}{2}\left[Y_{j 4}, Y_{j 6}\right]=Y_{46}, \frac{1}{2}\left[Y_{j 5}, Y_{j 6}\right]=Y_{56}, j=0,1,2,3, \\
& \frac{1}{2}\left[Y_{47}, Y_{57}\right]=Y_{45}, \frac{1}{2}\left[Y_{47}, Y_{67}\right]=Y_{46}, \frac{1}{2}\left[Y_{57}, Y_{67}\right]=Y_{56} .
\end{aligned}
$$

3. We aim to calculate the angles between the vector fields from $\mathcal{H}_{m}, m=$ $0,1,2,3,4$ and between vector fields from $\mathcal{H}_{m}$ and $V$. Beforehand, we introduce the following notations for the coordinates on the sphere $S^{4}$ given by the Hopf map $S^{3} \rightarrow S^{7} \rightarrow S^{4}$.

$$
\begin{aligned}
& a_{00}=y_{0}^{2}+y_{1}^{2}+y_{2}^{2}+y_{3}^{2}-y_{4}^{2}-y_{5}^{2}-y_{6}^{2}-y_{7}^{2}, \\
& a_{11}=2\left(y_{0} y_{4}+y_{1} y_{5}+y_{2} y_{6}+y_{3} y_{7}\right), \\
& a_{22}=2\left(-y_{0} y_{5}+y_{1} y_{4}-y_{2} y_{7}+y_{3} y_{6}\right), \\
& a_{33}=2\left(-y_{0} y_{6}+y_{1} y_{7}+y_{2} y_{4}-y_{3} y_{5}\right), \\
& a_{44}=2\left(-y_{0} y_{7}-y_{1} y_{6}+y_{2} y_{5}+y_{3} y_{4}\right) .
\end{aligned}
$$

The first index of $a_{m k}$ reflects the number of the collection $\mathcal{H}_{m}$, where they will appear and the second one is related to the number of the coordinate on $S^{4}$.

We start from $\mathcal{H}_{0}$ and calculate the inner products:

$$
\left\langle Y_{45}, Y_{67}\right\rangle=-\left\langle Y_{46}, Y_{57}\right\rangle=\left\langle Y_{56}, Y_{47}\right\rangle=a_{00}
$$

All other vector fields are orthogonal. We continue for $\mathcal{H}_{1}$.

$$
\begin{aligned}
& \left\langle Y_{45}, Y_{36}\right\rangle=-\left\langle Y_{46}, Y_{35}\right\rangle=\left\langle Y_{56}, Y_{34}\right\rangle=a_{11} \\
& \left\langle Y_{45}, Y_{37}\right\rangle=2\left(-y_{0} y_{5}+y_{1} y_{4}+y_{2} y_{7}-y_{3} y_{6}\right)=a_{12} \\
& \left\langle Y_{46}, Y_{37}\right\rangle=2\left(-y_{0} y_{6}-y_{1} y_{7}+y_{2} y_{4}+y_{3} y_{5}\right)=a_{13} \\
& \left\langle Y_{56}, Y_{37}\right\rangle=2\left(y_{0} y_{7}-y_{1} y_{6}+y_{2} y_{5}-y_{3} y_{4}\right)=a_{14} .
\end{aligned}
$$

All other vector fields in $\mathcal{H}_{1} \cup V$ are orthogonal. For the set $\mathcal{H}_{2}$ we see the following:

$$
\begin{aligned}
& -\left\langle Y_{45}, Y_{26}\right\rangle=\left\langle Y_{46}, Y_{25}\right\rangle=-\left\langle Y_{56}, Y_{24}\right\rangle=a_{22} \\
& \left\langle Y_{45}, Y_{27}\right\rangle=2\left(y_{0} y_{4}+y_{1} y_{5}-y_{2} y_{6}-y_{3} y_{7}\right)=a_{21} \\
& \left\langle Y_{46}, Y_{27}\right\rangle=2\left(-y_{0} y_{7}+y_{1} y_{6}+y_{2} y_{5}-y_{3} y_{4}\right)=a_{24} \\
& \left\langle Y_{56}, Y_{27}\right\rangle=2\left(-y_{0} y_{6}-y_{1} y_{7}-y_{2} y_{4}-y_{3} y_{5}\right)=a_{23}
\end{aligned}
$$

The other products between vector fields from $\mathcal{H}_{2} \cup V$ vanish. For $\mathcal{H}_{3}$ the situation is similar.

$$
\begin{aligned}
& \left\langle Y_{45}, Y_{16}\right\rangle=-\left\langle Y_{46}, Y_{15}\right\rangle=\left\langle Y_{56}, Y_{14}\right\rangle=a_{33} \\
& \left\langle Y_{45}, Y_{17}\right\rangle=2\left(-y_{0} y_{7}-y_{1} y_{6}-y_{2} y_{5}-y_{3} y_{4}\right)=a_{34} \\
& \left\langle Y_{46}, Y_{17}\right\rangle=2\left(-y_{0} y_{4}+y_{1} y_{5}-y_{2} y_{6}+y_{3} y_{7}\right)=a_{31} \\
& \left\langle Y_{56}, Y_{17}\right\rangle=2\left(-y_{0} y_{5}-y_{1} y_{4}+y_{2} y_{7}+y_{3} y_{6}\right)=a_{32}
\end{aligned}
$$


All other vector fields from $\mathcal{H}_{3} \cup V$ are orthogonal. For the last collection $\mathcal{H}_{4}$ we obtain.

$$
\begin{aligned}
& \left\langle Y_{45}, Y_{06}\right\rangle=-\left\langle Y_{46}, Y_{05}\right\rangle=\left\langle Y_{56}, Y_{04}\right\rangle=a_{44} \\
& \left\langle Y_{45}, Y_{07}\right\rangle=2\left(y_{0} y_{6}-y_{1} y_{7}+y_{2} y_{4}-y_{3} y_{5}\right)=a_{43} \\
& \left\langle Y_{46}, Y_{07}\right\rangle=2\left(-y_{0} y_{5}-y_{1} y_{4}-y_{2} y_{7}-y_{3} y_{6}\right)=a_{42} \\
& \left\langle Y_{56}, Y_{07}\right\rangle=2\left(y_{0} y_{4}-y_{1} y_{5}-y_{2} y_{6}+y_{3} y_{7}\right)=a_{41}
\end{aligned}
$$

with the rest of the product vanishing.

We notice some relations between the coefficients $a_{m k}$. The coordinates on $S^{4}$ possesses the equality

$$
a_{00}^{2}+a_{11}^{2}+a_{22}^{2}+a_{33}^{2}+a_{44}^{2}=1 .
$$

The direct calculations also show

$$
\begin{aligned}
& a_{00}^{2}+a_{11}^{2}+a_{12}^{2}+a_{13}^{2}+a_{14}^{2}=1 \\
& a_{00}^{2}+a_{21}^{2}+a_{22}^{2}+a_{23}^{2}+a_{24}^{2}=1 \\
& a_{00}^{2}+a_{31}^{2}+a_{32}^{2}+a_{33}^{2}+a_{34}^{2}=1 \\
& a_{00}^{2}+a_{41}^{2}+a_{42}^{2}+a_{43}^{2}+a_{44}^{2}=1 .
\end{aligned}
$$

In other words the sum of the squares of the cosines between vector fields from $\mathcal{H}_{m} \cup V, m=1,2,3,4$ is equal to $1-a_{00}^{2}$. Let us consider 2 cases: $0<a_{00}^{2} \leq 1$ and $a_{00}^{2}=0$.

CASE $0<a_{00}^{2} \leq 1$. This case corresponds to any point on $S^{4}$ except of the set

$$
S_{1}=\left\{y_{0}^{2}+y_{1}^{2}+y_{2}^{2}+y_{3}^{2}=y_{4}^{2}+y_{5}^{2}+y_{6}^{2}+y_{7}^{2}=1 / 2\right\} .
$$

We observe that the sum of the square of the cosines from (7.10):

$$
\sum_{k=1}^{4} a_{m k}^{2}=1-a_{00}^{2}, \quad m=1,2,3,4
$$

belongs to the interval $(0,1)$ and no one of the cosines can be equal to 1 . We conclude that each of $\mathcal{H}_{m}, m=1,2,3,4$, is transverse to $V$. Particularly, if $a_{00}^{2}=1$ then $\sum_{k=1}^{4} a_{m k}^{2}=0$ and $\mathcal{H}_{m} \perp V$. The latter situation occurs in the antipodal points $( \pm 1,0,0,0,0) \in S^{4}$ or is to say on the set

$$
\begin{aligned}
S_{2}= & \left\{y_{0}^{2}+y_{1}^{2}+y_{2}^{2}+y_{3}^{2}=0, y_{4}^{2}+y_{5}^{2}+y_{6}^{2}+y_{7}^{2}=1\right\} \\
& \bigcup\left\{y_{0}^{2}+y_{1}^{2}+y_{2}^{2}+y_{3}^{2}=1, y_{4}^{2}+y_{5}^{2}+y_{6}^{2}+y_{7}^{2}=0\right\} \in S^{7} .
\end{aligned}
$$

We also can consider a collection $\mathcal{H}_{0}$, as a possible horizontal bracket generating distribution, if we choose an adequate vector field $W$. If $a_{00} \in(0,1)$ 
we have

$$
0<a_{41}^{2}+a_{42}^{2}+a_{43}^{2}+a_{44}^{2}=1-a_{00}^{2}<1
$$

and none of the products in (7.8) can give 1. We conclude that $Y_{07}$ can not be collinear to $V=\left\{Y_{45}, Y_{46}, Y_{56}\right\}$. Therefore, we choose $W=Y_{07}$. By the same reason we could take $Y_{j 7}, j=1,2,3$. In the case when $a_{00}^{2}=1$ the vector fields $Y_{j 7}, j=0,1,2,3$ are orthogonal to $V$ from $(7.5)-(7.8)$ but $\mathcal{H}_{0}$ is collinear to $V$ from (7.4) and the collection $\mathcal{H}_{0}$ with $W=Y_{j 7}$ is not transverse to $V$.

CASE $a_{00}^{2}=0$. In this case the distribution $\mathcal{H}_{0}$ nicely serves as a bracket generating if we find a suitable vector field $W$. Notice that (7.9) becomes

$$
a_{11}^{2}+a_{22}^{2}+a_{33}^{2}+a_{44}^{2}=1 .
$$

The $a_{m m}$ can not vanish simultaneously. Without loss of generality, we can assume that $a_{44} \neq 0$. Then $a_{41}^{2}+a_{42}^{2}+a_{43}^{2}=1-a_{44}^{2}<1$ from (7.10) and the products (7.8) imply that $Y_{07}$ is transverse to $V$ and can be used as a vector field $W$. In the case $a_{44}^{2}=1$ we get that $Y_{07}$ is orthogonal to $V$. Since $W \perp Y_{j 7}, j=4,5,6$ the collection $\mathcal{H}_{0}$ with any choice of $Y_{j 7}, j=0, \ldots, 3$ will be orthonormal.

We formulate the latter result in the following theorem

Theorem 5. Let (7.1) be the quaternionic Hopf map with the vertical space

$$
V=\left\{Y_{45}, Y_{46}, Y_{56}\right\}
$$

$S_{1}$ and $S_{2}$ are given by (7.11) and (7.12). Then the Hopf map produces the following Ehresmann connection $\mathcal{H}_{p}, p \in S^{7}$ :

(i) if $p \notin S_{1}$ then $\mathcal{H}_{p}=\left(\mathcal{H}_{m}\right)_{p}$, for any choice of $m=1,2,3,4$;

(ii) if $p \notin S_{2}$ then $\mathcal{H}_{p}=\left(Y_{47}, Y_{57}, Y_{67}, Y_{j 7}\right)_{p}$, for any choice of $j=0,1,2,3$; and we have respectively

(i) $\operatorname{span}\left\{\left(\mathcal{H}_{m}\right)\right\}_{p} \oplus \operatorname{span}\{V\}_{p}=T_{p} S^{7}, m=1,2,3,4 \quad$ for $\quad p \in S^{7} \backslash S_{1}$.

(ii) $\operatorname{span}\left\{Y_{47}, Y_{57}, Y_{67}, Y_{j 7}\right\}_{p} \oplus \operatorname{span}\{V\}_{p}=T_{p} S^{7}, j=0,1,2,3$, for $p \in$ $S^{7} \backslash S_{2}$.

Remark: During the referee process, we were pointed out of the paper [4] where a globally defined basis of the horizontal distribution of rank 4 was constructed considering the Clifford algebra structure of $S^{7}$. However, in this case a globally defined basis of the vertical space was not found. In our case, we present a globally defined basis of right invariant vector fields of the vertical space that correspond to the Lie algebra $\mathfrak{s u}(2)$ of the $S^{3}$-bundle. Nevertheless we did not succeeded in constructing a globally defined basis for the horizontal distribution. The question if both the horizontal distribution and the $S^{3}$-fiber are trivializable, remains open. 


\section{Appendix}

\subsection{Multiplication of octonions}

Let

$$
\begin{aligned}
& o_{1}=\left(x_{0} e_{0}+x_{1} e_{1}+x_{2} e_{2}+x_{3} e_{3}+x_{4} e_{4}+x_{5} e_{5}+x_{6} e_{6}+x_{7} e_{7}\right) \quad \text { and } \\
& o_{2}=\left(y_{0} e_{0}+y_{1} e_{1}+y_{2} e_{2}+y_{3} e_{3}+y_{4} e_{4}+y_{5} e_{5}+y_{6} e_{6}+y_{7} e_{7}\right)
\end{aligned}
$$

be two octonions. Then we have according to Table 1

$$
\begin{aligned}
o_{1} \cdot o_{2}= & \left(x_{0} e_{0}+x_{1} e_{1}+x_{2} e_{2}+x_{3} e_{3}+x_{4} e_{4}+x_{5} e_{5}+x_{6} e_{6}+x_{7} e_{7}\right) \\
& \circ\left(y_{0} e_{0}+y_{1} e_{1}+y_{2} e_{2}+y_{3} e_{3}+y_{4} e_{4}+y_{5} e_{5}+y_{6} e_{6}+y_{7} e_{7}\right) \\
= & \left(x_{0} y_{0}-x_{1} y_{1}-x_{2} y_{2}-x_{3} y_{3}-x_{4} y_{4}-x_{5} y_{5}-x_{6} y_{6}-x_{7} y_{7}\right) e_{0} \\
& +\left(x_{1} y_{0}+x_{0} y_{1}-x_{3} y_{2}+x_{2} y_{3}-x_{5} y_{4}+x_{4} y_{5}+x_{7} y_{6}-x_{6} y_{7}\right) e_{1} \\
& +\left(x_{2} y_{0}+x_{3} y_{1}+x_{0} y_{2}-x_{1} y_{3}-x_{6} y_{4}-x_{7} y_{5}+x_{4} y_{6}+x_{5} y_{7}\right) e_{2} \\
& +\left(x_{3} y_{0}-x_{2} y_{1}+x_{1} y_{2}+x_{0} y_{3}-x_{7} y_{4}+x_{6} y_{5}-x_{5} y_{6}+x_{4} y_{7}\right) e_{3} \\
& +\left(x_{4} y_{0}+x_{5} y_{1}+x_{6} y_{2}+x_{7} y_{3}+x_{0} y_{4}-x_{1} y_{5}-x_{2} y_{6}-x_{3} y_{7}\right) e_{4} \\
& +\left(x_{5} y_{0}-x_{4} y_{1}+x_{7} y_{2}-x_{6} y_{3}+x_{1} y_{4}+x_{0} y_{5}+x_{3} y_{6}-x_{2} y_{7}\right) e_{5} \\
& +\left(x_{6} y_{0}-x_{7} y_{1}-x_{4} y_{2}+x_{5} y_{3}+x_{2} y_{4}-x_{3} y_{5}+x_{0} y_{6}+x_{1} y_{7}\right) e_{6} \\
& +\left(x_{7} y_{0}+x_{6} y_{1}-x_{5} y_{2}-x_{4} y_{3}+x_{3} y_{4}+x_{2} y_{5}-x_{1} y_{6}+x_{0} y_{7}\right) e_{7} .
\end{aligned}
$$

\subsection{Vector fields}

According to the previous multiplication rule, we have the following unit vector fields of $\mathbb{R}^{8}$ arising as right invariant vector fields under the octonion product.

$$
\begin{aligned}
& Y_{0}(y)=y_{0} \partial_{y_{0}}+y_{1} \partial_{y_{1}}+y_{2} \partial_{y_{2}}+y_{3} \partial_{y_{3}}+y_{4} \partial_{y_{4}}+y_{5} \partial_{y_{5}}+y_{6} \partial_{y_{6}}+y_{7} \partial_{y_{7}} \\
& Y_{1}(y)=-y_{1} \partial_{y_{0}}+y_{0} \partial_{y_{1}}-y_{3} \partial_{y_{2}}+y_{2} \partial_{y_{3}}-y_{5} \partial_{y_{4}}+y_{4} \partial_{y_{5}}-y_{7} \partial_{y_{6}}+y_{6} \partial_{y_{7}} \\
& Y_{2}(y)=-y_{2} \partial_{y_{0}}+y_{3} \partial_{y_{1}}+y_{0} \partial_{y_{2}}-y_{1} \partial_{y_{3}}-y_{6} \partial_{y_{4}}+y_{7} \partial_{y_{5}}+y_{4} \partial_{y_{6}}-y_{5} \partial_{y_{7}} \\
& Y_{3}(y)=-y_{3} \partial_{y_{0}}-y_{2} \partial_{y_{1}}+y_{1} \partial_{y_{2}}+y_{0} \partial_{y_{3}}+y_{7} \partial_{y_{4}}+y_{6} \partial_{y_{5}}-y_{5} \partial_{y_{6}}-y_{4} \partial_{y_{7}} \\
& Y_{4}(y)=-y_{4} \partial_{y_{0}}+y_{5} \partial_{y_{1}}+y_{6} \partial_{y_{2}}-y_{7} \partial_{y_{3}}+y_{0} \partial_{y_{4}}-y_{1} \partial_{y_{5}}-y_{2} \partial_{y_{6}}+y_{3} \partial_{y_{7}} \\
& Y_{5}(y)=-y_{5} \partial_{y_{0}}-y_{4} \partial_{y_{1}}-y_{7} \partial_{y_{2}}-y_{6} \partial_{y_{3}}+y_{1} \partial_{y_{4}}+y_{0} \partial_{y_{5}}+y_{3} \partial_{y_{6}}+y_{2} \partial_{y_{7}} \\
& Y_{6}(y)=-y_{6} \partial_{y_{0}}+y_{7} \partial_{y_{1}}-y_{4} \partial_{y_{2}}+y_{5} \partial_{y_{3}}+y_{2} \partial_{y_{4}}-y_{3} \partial_{y_{5}}+y_{0} \partial_{y_{6}}-y_{1} \partial_{y_{7}} \\
& Y_{7}(y)=-y_{7} \partial_{y_{0}}-y_{6} \partial_{y_{1}}+y_{5} \partial_{y_{2}}+y_{4} \partial_{y_{3}}-y_{3} \partial_{y_{4}}-y_{2} \partial_{y_{5}}+y_{1} \partial_{y_{6}}+y_{0} \partial_{y_{7}} .
\end{aligned}
$$

The vector fields $Y_{i}, i=1, \ldots, 7$ form an orthonormal frame of $T_{p} S^{7}$, $p \in S^{7}$, with respect to restriction of the inner product $\langle\cdot, \cdot\rangle$ from $\mathbb{R}^{8}$ to the tangent space $T_{p} S^{7}$ at each $p \in S^{7}$. 


\subsection{Commutators between vector fields}

Let us denote by $Y_{i j}(y)=\frac{1}{2}\left[Y_{i}(y), Y_{j}(y)\right]$ the commutator between the right invariant vector fields under the octonion product, described in the previous Subsection, we have the following list:

$$
\begin{aligned}
& Y_{12}(y)=y_{3} \partial_{y_{0}}+y_{2} \partial_{y_{1}}-y_{1} \partial_{y_{2}}-y_{0} \partial_{y_{3}}+y_{7} \partial_{y_{4}}+y_{6} \partial_{y_{5}}-y_{5} \partial_{y_{6}}-y_{4} \partial_{y_{7}} \\
& Y_{13}(y)=-y_{2} \partial_{y_{0}}+y_{3} \partial_{y_{1}}+y_{0} \partial_{y_{2}}-y_{1} \partial_{y_{3}}+y_{6} \partial_{y_{4}}-y_{7} \partial_{y_{5}}-y_{4} \partial_{y_{6}}+y_{5} \partial_{y_{7}} \\
& Y_{14}(y)=y_{5} \partial_{y_{0}}+y_{4} \partial_{y_{1}}-y_{7} \partial_{y_{2}}-y_{6} \partial_{y_{3}}-y_{1} \partial_{y_{4}}-y_{0} \partial_{y_{5}}+y_{3} \partial_{y_{6}}+y_{2} \partial_{y_{7}} \\
& Y_{15}(y)=-y_{4} \partial_{y_{0}}+y_{5} \partial_{y_{1}}-y_{6} \partial_{y_{2}}+y_{7} \partial_{y_{3}}+y_{0} \partial_{y_{4}}-y_{1} \partial_{y_{5}}+y_{2} \partial_{y_{6}}-y_{3} \partial_{y_{7}} \\
& Y_{16}(y)=y_{7} \partial_{y_{0}}+y_{6} \partial_{y_{1}}+y_{5} \partial_{y_{2}}+y_{4} \partial_{y_{3}}-y_{3} \partial_{y_{4}}-y_{2} \partial_{y_{5}}-y_{1} \partial_{y_{6}}-y_{0} \partial_{y_{7}} \\
& Y_{17}(y)=-y_{6} \partial_{y_{0}}+y_{7} \partial_{y_{1}}+y_{4} \partial_{y_{2}}-y_{5} \partial_{y_{3}}-y_{2} \partial_{y_{4}}+y_{3} \partial_{y_{5}}+y_{0} \partial_{y_{6}}-y_{1} \partial_{y_{7}} \\
& Y_{23}(y)=y_{1} \partial_{y_{0}}-y_{0} \partial_{y_{1}}+y_{3} \partial_{y_{2}}-y_{2} \partial_{y_{3}}-y_{5} \partial_{y_{4}}+y_{4} \partial_{y_{5}}-y_{7} \partial_{y_{6}}+y_{6} \partial_{y_{7}} \\
& Y_{24}(y)=y_{6} \partial_{y_{0}}+y_{7} \partial_{y_{1}}+y_{4} \partial_{y_{2}}+y_{5} \partial_{y_{3}}-y_{2} \partial_{y_{4}}-y_{3} \partial_{y_{5}}-y_{0} \partial_{y_{6}}-y_{1} \partial_{y_{7}} \\
& Y_{25}(y)=-y_{7} \partial_{y_{0}}+y_{6} \partial_{y_{1}}+y_{5} \partial_{y_{2}}-y_{4} \partial_{y_{3}}+y_{3} \partial_{y_{4}}-y_{2} \partial_{y_{5}}-y_{1} \partial_{y_{6}}+y_{0} \partial_{y_{7}} \\
& Y_{26}(y)=-y_{4} \partial_{y_{0}}-y_{5} \partial_{y_{1}}+y_{6} \partial_{y_{2}}+y_{7} \partial_{y_{3}}+y_{0} \partial_{y_{4}}+y_{1} \partial_{y_{5}}-y_{2} \partial_{y_{6}}-y_{3} \partial_{y_{7}} \\
& Y_{27}(y)=y_{5} \partial_{y_{0}}-y_{4} \partial_{y_{1}}+y_{7} \partial_{y_{2}}-y_{6} \partial_{y_{3}}+y_{1} \partial_{y_{4}}-y_{0} \partial_{y_{5}}+y_{3} \partial_{y_{6}}-y_{2} \partial_{y_{7}} \\
& Y_{34}(y)=-y_{7} \partial_{y_{0}}+y_{6} \partial_{y_{1}}-y_{5} \partial_{y_{2}}+y_{4} \partial_{y_{3}}-y_{3} \partial_{y_{4}}+y_{2} \partial_{y_{5}}-y_{1} \partial_{y_{6}}+y_{0} \partial_{y_{7}} \\
& Y_{35}(y)=-y_{6} \partial_{y_{0}}-y_{7} \partial_{y_{1}}+y_{4} \partial_{y_{2}}+y_{5} \partial_{y_{3}}-y_{2} \partial_{y_{4}}-y_{3} \partial_{y_{5}}+y_{0} \partial_{y_{6}}+y_{1} \partial_{y_{7}} \\
& Y_{36}(y)=y_{5} \partial_{y_{0}}-y_{4} \partial_{y_{1}}-y_{7} \partial_{y_{2}}+y_{6} \partial_{y_{3}}+y_{1} \partial_{y_{4}}-y_{0} \partial_{y_{5}}-y_{3} \partial_{y_{6}}+y_{2} \partial_{y_{7}} \\
& Y_{37}(y)=y_{4} \partial_{y_{0}}+y_{5} \partial_{y_{1}}+y_{6} \partial_{y_{2}}+y_{7} \partial_{y_{3}}-y_{0} \partial_{y_{4}}-y_{1} \partial_{y_{5}}-y_{2} \partial_{y_{6}}-y_{3} \partial_{y_{7}} \\
& Y_{45}(y)=y_{1} \partial_{y_{0}}-y_{0} \partial_{y_{1}}-y_{3} \partial_{y_{2}}+y_{2} \partial_{y_{3}}+y_{5} \partial_{y_{4}}-y_{4} \partial_{y_{5}}-y_{7} \partial_{y_{6}}+y_{6} \partial_{y_{7}} \\
& Y_{46}(y)=y_{2} \partial_{y_{0}}+y_{3} \partial_{y_{1}}-y_{0} \partial_{y_{2}}-y_{1} \partial_{y_{3}}+y_{6} \partial_{y_{4}}+y_{7} \partial_{y_{5}}-y_{4} \partial_{y_{6}}-y_{5} \partial_{y_{7}} \\
& Y_{47}(y)=-y_{3} \partial_{y_{0}}+y_{2} \partial_{y_{1}}-y_{1} \partial_{y_{2}}+y_{0} \partial_{y_{3}}+y_{7} \partial_{y_{4}}-y_{6} \partial_{y_{5}}+y_{5} \partial_{y_{6}}-y_{4} \partial_{y_{7}} \\
& Y_{56}(y)=-y_{3} \partial_{y_{0}}+y_{2} \partial_{y_{1}}-y_{1} \partial_{y_{2}}+y_{0} \partial_{y_{3}}-y_{7} \partial_{y_{4}}+y_{6} \partial_{y_{5}}-y_{5} \partial_{y_{6}}+y_{4} \partial_{y_{7}} \\
& Y_{57}(y)=-y_{2} \partial_{y_{0}}-y_{3} \partial_{y_{1}}+y_{0} \partial_{y_{2}}+y_{1} \partial_{y_{3}}+y_{6} \partial_{y_{4}}+y_{7} \partial_{y_{5}}-y_{4} \partial_{y_{6}}-y_{5} \partial_{y_{7}} \\
& Y_{67}(y)=y_{1} \partial_{y_{0}}-y_{0} \partial_{y_{1}}-y_{3} \partial_{y_{2}}+y_{2} \partial_{y_{3}}-y_{5} \partial_{y_{4}}+y_{4} \partial_{y_{5}}+y_{7} \partial_{y_{6}}-y_{6} \partial_{y_{7}} \text {. }
\end{aligned}
$$

\section{References}

[1] Adams, J.F.: Vector fields on spheres. Ann. of Math. (2) 75 (1962), 603-632.

[2] Agrachev, A., Boscain, U., Gauthier, J.-P. and Rossi, F.: The intrinsic hypoelliptic Laplacian and its heat kernel on unimodular Lie groups. J. Funct. Anal. 256 (2009), no. 8, 2621-2655.

[3] Alekseevsky, D. and Kamishima, Y.: Pseudo-conformal quaternionic CR structure on $(4 n+3)$ - dimensional manifolds. Ann. Mat. Pura Appl. (4) 187 (2008), no. 3, 487-529. 
[4] Bauer, W. And Furutani, K.: Spectral analysis and geometry of a subRiemannian structure on $S^{3}$ and $S^{7}$. J. Geom. Phys. 58 (2008), no. 12, 1693-1738.

[5] Boggess, A.: CR manifolds and the tangential Cauchy-Riemann complex. Studies in Advanced Mathematics. CRC Press, Boca Raton, FL, 1991.

[6] Boscain, U. And Rossi, F.: Invariant Carnot-Caratheodory metrics on $S^{3}, S O(3), S L(2)$ and lens spaces. SIAM J. Control Optim. 47 (2008), no. $4,1851-1878$.

[7] Bott, R. And Milnor, J.: On the parallelizability of the spheres. Bull. Amer. Math. Soc. 64 (1958), 87-89.

[8] Cartan, H.: Differential forms. Dover Publications, New York, 2006.

[9] Chang, D.-C. And Markina, I.: Geometric analysis on quaternion $\mathbb{H}$-type groups. J. Geom. Anal. 16 (2006), no. 2, 265-294.

[10] Chang, D.-C., Markina, I. and Vasil'ev, A.: Sub-Riemannian geodesics on the 3-D sphere. Complex Anal. Oper. Theory 3 (2009), no. 2, 361-377.

[11] Chow, W.-L.: Über Systeme von linearen partiellen Differentialgleichungen erster Ordnung. Math. Ann. 117 (1939), 98-105.

[12] Darboux, G.: Sur le problème de Pfaff. Bull. Sci. Math. 6 (1882) 14-36, 49-68.

[13] Gaveau, B.: Principe de moindre action, propagation de la chaleur et estimées sous elliptiques sur certains groupes nilpotents. Acta Math. 139 (1977), no. 1-2, 95-153.

[14] Gershkovich, V. YA. And Vershik, A. M.: Geodesic flow on SL $(2, R)$ with nonholonomic constraints. Zap. Nauchn. Sem. Leningrad. Otdel. Mat. Inst. Steklov. (LOMI) 155 (1986), Differentsialnaya Geometriya, Gruppy Li i Mekh. VIII, 7-17, 193; translation in J. Soviet Math. 41 (1988), no. 2, 891-898.

[15] Gromov, M.: Carnot-Carathéodory spaces seen from within. In SubRiemannian geometry, 79-323. Prog. Math. 144. Birkhäuser, Basel, 1996.

[16] Hopf, H.: Über die Abbildungen von Sphären auf Sphären niedrigerer Dimension Math. Ann. 104 (1931), 637-665.

[17] Hurtado, A. and Rosales, C.: Area-stationary surfaces inside the subRiemannian three-sphere. Math. Ann. 340 (2008), no. 3, 675-708.

[18] Liu, W. And Sussmann, H. J.: Shortest paths for sub-Riemannian metrics on rank-two distributions. Mem. Amer. Math. Soc. 118 (1995), no. 564, 104 pp.

[19] Lyons, D. W.: An elementary introduction to the Hopf fibration. Math. Mag. 76 (2003), no. 2, 87-98.

[20] Margulis, G. and Mostow, G.: Some remarks on the definition of tangent cones in a Carnot-Carathéodory space. J. Anal. Math. 80 (2000), $299-317$. 
[21] Mitchell, J.: On Carnot-Carathéodory metrics. J. Differential Geom. 21 (1985), no. 1, 35-45.

[22] Montgomery, R.: A tour of subriemannian geometries, their geodesics and applications. Mathematical Surveys and Monographs 91. American Mathematical Society, Providence, RI, 2002.

[23] Rashevskil, P. K.: About connecting two points of complete nonholonomic space by admissible curve. Uch. Zapiski Ped. Inst. K. Liebknecht 2 (1938), 83-94.

[24] Strichartz, R.: Sub-Riemannian geometry. J. Differential Geom. 24 (1986), no. 2, 221-263.

[25] Sussmann, H.: Orbits of families of vector fields and integrability of distributions. Trans. Amer. Math. Soc. 180 (1973), 171-188.

[26] Urbantke, H. K.: The Hopf fibration-seven times in physics. J. Geom. Phys. 46 (2003), no. 2, 125-150.

[27] VICK, J.: Homology theory. An introduction to algebraic topology. Graduate Texts in Mathematics 145. Springer-Verlag, New York, 1994.

Recibido: 16 de mayo de 2009

Mauricio Godoy Molina

Department of Mathematics

University of Bergen

Norway

mauricio.godoy@math.uib.no

Irina Markina

Department of Mathematics

University of Bergen

Norway

irina.markina@uib.no

The authors are partially supported by the grant of the Norwegian Research Council \# 204726/V30 and by the grant of the European Science Foundation Networking Programme HCAA. 